\title{
Efficacy of intestinal obstruction stent combined with laparoscopic surgery and neoadjuvant chemotherapy in patients with obstructive colorectal cancer
}

\author{
LEILEI YANG $^{1 *}$, WEIWEI MA ${ }^{2 *}$, MEIZHEN WANG $^{3}$, RUILI ZHANG $^{1}$, TIENAN BI $^{1}$ and SHENKANG ZHOU ${ }^{1}$ \\ Departments of ${ }^{1}$ Gastrointestinal Surgery and ${ }^{2}$ Endoscopic Center and ${ }^{3}$ Surgical Department, \\ Taizhou Hospital of Zhejiang Province, Wenzhou Medical University, Taizhou, Zhejiang 317000, P.R. China
}

Received September 4, 2018; Accepted June 6, 2019

DOI: $10.3892 / \mathrm{ol} .2019 .10525$

\begin{abstract}
There is still controversy on the surgical treatment of obstructive colorectal cancer worldwide. No accurate research has been reported to propose which method is the most suitable for patients with obstructive colorectal cancer. Therefore, comparison of efficacy of intestinal stent and trans-anal ileus catheter combined with laparoscopic surgery and neoadjuvant chemotherapy respectively in patients with obstructive colorectal cancer was carried out to provide reference and guidance for the selection of surgical schemes for patients with obstructive colorectal cancer. In total 89 patients with obstructive colorectal cancer treated in the Taizhou Hospital of Zhejiang Province, Wenzhou Medical University, from February 2016 to March 2017 were selected for retrospective analysis. Forty-nine cases treated with intestinal metal stent implantation combined with laparoscopic surgery and neoadjuvant chemotherapy were the stent group. The other 40 cases treated with trans-anal ileus catheter combined with laparoscopic surgery and neoadjuvant chemotherapy were the catheter group. The intestinal preparation time, surgical duration, intraoperative blood loss, open surgery rate, postoperative exhaust time and adverse reaction rate were compared between the two groups. All the patients were followed up with reexamination at 1 year in hospital to record the local recurrence rate and tumor implantation rate of incision. The intestinal preparation time in the stent group was shorter than that in the catheter group $(\mathrm{P}<0.001)$. The surgical duration in the stent group was longer than that in the catheter group $(\mathrm{P}<0.001)$. The intraoperative blood loss in the stent group was higher
\end{abstract}

Correspondence to: $\mathrm{Dr}$ Shenkang Zhou, Department of Gastrointestinal Surgery, Taizhou Hospital of Zhejiang Province, Wenzhou Medical University, 150 Ximen Street, Taizhou, Zhejiang 317000, P.R. China

E-mail: 13736249308@163.com

${ }^{*}$ Contributed equally

Key words: intestinal obstruction stent, Tran-sanal ileus catheter, obstructive colorectal cancer, laparoscopy, neoadjuvant chemotherapy than that in the catheter group $(\mathrm{P}<0.001)$. However, there was no significant difference in open surgery rate, postoperative exhaust time, adverse reaction rate, local recurrence rate or incision tumor implantation rate between the two groups (all $\mathrm{P}>0.05)$. Therefore, intestinal metal stent implantation can effectively relieve intestinal obstruction, while trans-anal ileus catheter has higher safety in laparoscopic surgery. Their combination with neoadjuvant chemotherapy and laparoscopic surgery for obstructive colorectal cancer has high value and clinical effect. The best treatment plan should be selected according to the patient's condition.

\section{Introduction}

Colorectal cancer is the most common malignant tumor in the digestive tract and has a high incidence (1). According to statistics, more than 8 million new colorectal cancer cases were reported worldwide in 2016, and the cumulative number has reached $\sim 300$ million (2). In colorectal cancer patients, intestinal obstruction is a very common tumor complication, and there are studies indicating that the risk of colorectal cancer patients with intestinal obstruction is as high as $10-30 \%$ (3). The body of patients with colon cancer is in a very weak state, the occurrence of intestinal obstruction at this time causes acid-base imbalance, water and electrolyte disorders, and intestinal surgery cannot be performed routinely (4). Edema and hyperemia are common in the intestinal tract of obstruction segment. Patients with acute tumor condition can only be operated by stages, which not only increases the risk and cost of surgery, but also has a great negative effect on the prognosis of patients (5). According to statistics, the effective cure rate of colorectal cancer patients with intestinal obstruction is only $60-80 \%$ (6) and intestinal obstruction is one of the main reasons for the poor prognosis of colorectal cancer patients. In the face of this situation, continuous research and development of colorectal cancer in clinic can effectively improve the prognosis of patients with intestinal obstruction. With the application of neoadjuvant chemotherapy, the tumor focus is first reduced by chemotherapy, and then resected by surgery, which can significantly improve the curative effect of the patients (7). Trans-anal ileus catheter and intestinal metal stent implantation are the latest laparoscopic surgery adjuvant 
therapy, which can not only effectively improve the tumor resection rate, but also greatly increase the rate of primary intestinal anastomosis and they have been proved to have a very high application value in obstructive colorectal cancer (8-10). However, the application of trans-anal ileus catheter and intestinal metal stent implantation in obstructive colorectal cancer worldwide is mostly used as guidance study $(11,12)$, and a relatively rare comparison exists of the exact curative effect between them. Since 2016, Taizhou Hospital has popularized the use of trans-anal ileus catheter, intestinal metal stent implantation combined with cancer laparoscopic surgery and neoadjuvant chemotherapy in the treatment of obstructive colorectal cancer patients, and has achieved significant results. There is still controversy on surgical treatment of obstructive colorectal cancer worldwide. To the best of our knowledge, there is no accurate research to propose which method is most suitable for patients with obstructive colorectal cancer. Therefore, this study compared the clinical effects of intestinal stent and trans-anal ileus catheter combined with laparoscopic surgery and neoadjuvant chemotherapy respectively in patients with obstructive colorectal cancer to provide reference and guidance for the selection of surgical schemes for patients with obstructive colorectal cancer.

\section{Patients and methods}

General data. Patients with obstructive colorectal cancer treated in Taizhou Hospital of Zhejiang Province, Wenzhou Medical University (Taizhou, China), from February 2016 to March 2017 were selected for retrospective analysis. Inclusion criteria: Patients in accordance with the clinical manifestations of colorectal cancer and highly suspected as colorectal cancer in Taizhou Hospital of Zhejiang Province, Wenzhou Medical University (13), after pathological biopsy, confirmed as colorectal cancer with intestinal obstruction. Patients had trans-anal ileus catheter and intestinal metal stent implantation combined with laparoscopic surgery and neoadjuvant chemotherapy in the hospital after diagnosis. Patients aged 30 to 70 years; patients who were willing to cooperate with the medical staff, and patients with complete records. Exclusion criteria: Patients with other tumors; patients with cardiovascular and cerebrovascular diseases; patients with severe cardiopulmonary dysfunction; pregnant patients; patients with chemotherapy tolerance; patients with physical disabilities; long-term bedridden patients; patients with mental illness; patients transferred to hospital in midway; patients who had received radiotherapy and chemotherapy before admission. In total, 89 cases were selected, including 67 males and 22 females with an age range of 38-67 years and an average age of $51.69 \pm 10.77$ years. Forty-nine of 89 patients treated with intestinal metal stent implantation combined with laparoscopic surgery and neoadjuvant chemotherapy were the stent group. The other 40 cases treated with trans-anal ileus catheter combined with laparoscopic surgery and neoadjuvant chemotherapy were the catheter group.

This study was approved by the Ethics Committee of Taizhou Hospital of Zhejiang Province, Wenzhou Medical University. Patients who participated in this research, signed an informed consent and had complete clinical data.

\section{Methods}

Study design. Two groups of patients underwent intestinal obstruction surgery followed by tumor resection and chemotherapy after surgery. The clinical efficacy and prognosis of the two groups were compared after all courses of treatment.

Surgical protocol in the stent group. The patients were placed in a left lateral decubitus position. When the enteroscope entered the body and reached the stenotic part, an expansion catheter was used to send the guide wire into the stenotic part under X-ray monitoring. Appropriate stent was chosen according to the length and width of the tumor invading intestine (NDS-20-080-230; Beijing YuLongKeTai Trading Co., Ltd., Beijing, China). A stent pusher was used to drive the stent along the wire to the stenotic part, both ends exceeding the narrow point at least by $2 \mathrm{~cm}$. The changes of vital signs were closely monitored after stent placement.

Surgical protocol in the catheter group. Endoscopy entered into the obstruction for angiography after lavage of obstruction of the distal intestinal. If the contrast agent was unable to pass through the narrow area, the catheter placement was abandoned, if it passed, the guide wire was inserted from the biopsy hole, and through the narrow part under X-ray monitoring. A clamp expander was inserted along the guide wire and the enteroscope was pulled out. The intestinal obstruction catheter (D1407-0518; Dalian Create Medic International Trade Co., Ltd.) was inserted into the airbag near the narrow area along the clamp expander, and the guide wire and the clamp expander were pulled out to complete the catheter placement. The vital signs of the patients were closely monitored after catheterization, and warm saline $1000-1500 \mathrm{ml}$ was used daily to wash the obstruction catheter.

Laparoscopic surgery. All the patients were operated by physicians with secondary senior professional title or above in Oncological Surgery of the hospital. The stents in the stent group were removed with tumor resection during surgery, and the catheters in the catheter group were removed before the surgery.

Neoadjuvant chemotherapy regimen. MFOLFOX6 protocol was used, including oxaliplatin (EB01714; Shanghai Shifeng Biological Technology Co., Ltd.) $85 \mathrm{mg} / \mathrm{m}^{2}$, intravenous drip, $d_{1}$. Calcium folinate (RB768; Shanghai GuangRui Biological Technology Co., Ltd.) $200 \mathrm{mg} / \mathrm{m}^{2}$, intravenous drip, $\mathrm{d}_{1}$. Fluorouracil for injection (0597-5G; Qingdao Jisskang Biotechnology Co., Ltd.) $400 \mathrm{mg} / \mathrm{m}^{2}$, venous injection, $\mathrm{d}_{1}$. Then fluorouracil $2.0 \mathrm{~g} / \mathrm{m}^{2}$ was added to the chemotherapeutic pump for $48 \mathrm{~h}$ intravenous drip, repeated every 2 weeks, and the duration of chemotherapy was 2-3 months.

Observation indicators. Preoperative indicators: Clinical information such as sex, age, tumor stage and differentiation degree of patients in the two groups. Intraoperative indicators: The intestinal preparation time, surgical duration, intraoperative blood loss and open surgery rate (open surgery was performed when the tumor was not completely resected by laparoscopic surgery) of patients in the two groups were observed. Postoperative indicators: postoperative exhaust time and adverse reaction rate (including postoperative anastomotic fistula, incision infection and lower extremity venous thrombosis). Prognostic indicators: All the patients were included in prognostic follow-up of reexamination at 1 year in hospital to 

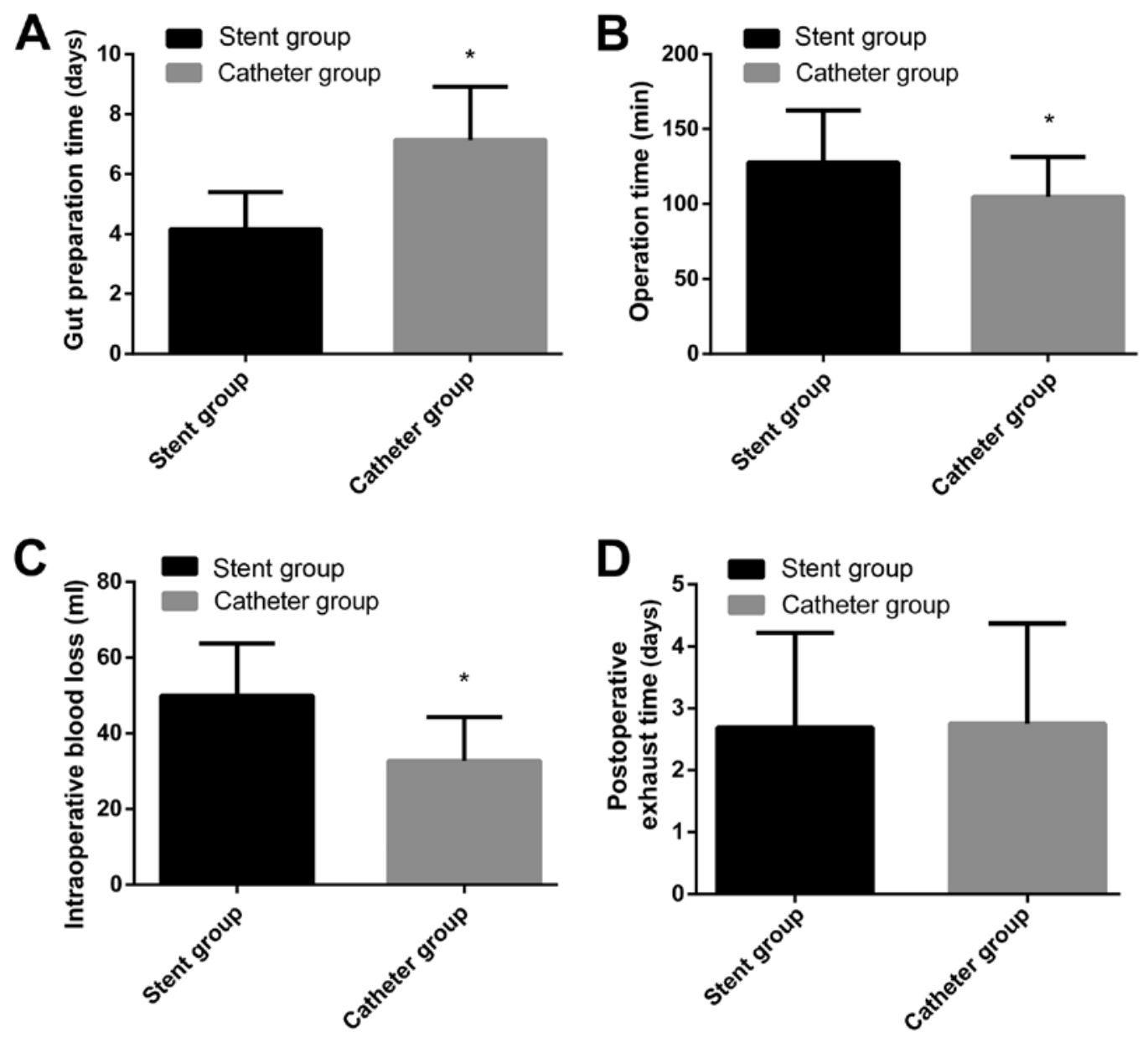

Figure 1. (A) Comparison of the intestinal preparation time between the two groups. The intestinal preparation time in the catheter group was significantly longer than that in the stent group; ${ }^{*} \mathrm{P}<0.001$, compared with the intestinal preparation time in the stent group. (B) Comparison of surgical duration between the two groups. The surgical duration in the catheter group was significantly shorter than that in the stent group; ${ }^{*} \mathrm{P}<0.001$, compared with the surgical duration in the stent group. (C) Comparison of intraoperative blood loss between the two groups. The intraoperative blood loss in the catheter group was significantly less than that in the stent group; ${ }^{*}<0.001$, compared with the intraoperative blood loss in the stent group. (D) Comparison of postoperative exhaust time between the two groups. There was no significant difference in postoperative exhaust time between the catheter and and stent groups; P $>0.05$.

record the local recurrence rate and incision tumor implantation rate.

Statistical analysis. The data were analyzed and processed by SPSS 24.0 statistical software (Beijing Bizinsight Information Technology Co., Ltd.). The enumeration data were expressed in the form of a rate. Chi-square test was used for comparison between the groups. The measured data were expressed as mean \pm standard deviation, and t-test was used for comparison between the two groups. $\mathrm{P}<0.05$ was considered statistically significant.

\section{Results}

Preoperative indicators. There was no significant difference in age, body weight, disease course, the count of platelet, erythrocytes and leukocytes, time interval after stent/tube insertion, sex, smoking, alcohol consumption, exercise habit, ethnicity, pathological stage, lymphatic metastasis, differentiation, intestinal obstruction degree, primary tumor, histological type and lymph node dissection between the two groups $(\mathrm{P}>0.05)$, which proved that the two groups were comparable (Table I).
Intraoperative indicators. The intestinal preparation time in the stent group $(4.16 \pm 1.24$ days $)$ was shorter than that in the catheter group $(7.14 \pm 1.78$ days $)(\mathrm{P}<0.001)$; The surgical duration in the stent group $(127.68 \pm 34.74 \mathrm{~min})$ was longer than that in the catheter group $(104.67 \pm 26.77 \mathrm{~min})(\mathrm{P}<0.001)$. The intraoperative blood loss in the stent group $(49.87 \pm 13.81 \mathrm{ml})$ was higher than that in the catheter group $(32.73 \pm 11.57 \mathrm{ml})$ $(\mathrm{P}<0.001)$ (Fig. 1A-C). Two patients underwent open surgery in the stent group, and the open surgery rate was $4.08 \%$; 3 patients underwent open surgery in the catheter group, and the open surgery rate was $7.50 \%$. There was no significant difference in the open surgery rate between the two groups (P>0.05) (Table II).

Postoperative indicators. The postoperative exhaust time in the stent group was $2.69 \pm 1.53$ days, which was not significantly different from that in the catheter group $(2.75 \pm 1.62$ days $)$ ( $\mathrm{P}>0.05)$. In the stent group, 6.12\% (3 cases) of patients had incision infection; $2.04 \%$ (1 case) had anastomotic fistula; $2.04 \%$ ( 1 case) had lower extremity venous thrombosis; $4.08 \%$ ( 2 cases) had abdominal infection; $8.16 \%$ ( 4 cases) had abdominal pain; $6.12 \%$ ( 3 cases) had fever, and the incidence of adverse reactions was $28.57 \%$ (14 cases). In the catheter 
Table I. Clinical data comparison [n (\%)].

\begin{tabular}{|c|c|c|c|c|}
\hline Factors & Stent group $(\mathrm{n}=49)$ & Catheter group $(n=40)$ & $\chi^{2}$ or t-test & P-value \\
\hline Age & $50.64 \pm 11.57$ & $51.04 \pm 10.62$ & 0.168 & 0.867 \\
\hline Body weight $(\mathrm{kg})$ & $76.24 \pm 8.67$ & $75.14 \pm 8.32$ & 0.606 & 0.546 \\
\hline BMI $\left(\mathrm{kg} / \mathrm{m}^{2}\right)$ & $26.13 \pm 4.38$ & $27.05 \pm 5.15$ & 0.911 & 0.365 \\
\hline Disease course (weeks) & $3.17 \pm 0.54$ & $3.22 \pm 0.50$ & 0.449 & 0.655 \\
\hline Platelet $\left(\times 10^{9} / 1\right)$ & $228.21 \pm 50.14$ & $220.42 \pm 57.21$ & 0.684 & 0.684 \\
\hline Erythrocyte $\left(\times 10^{9} / 1\right)$ & $4.66 \pm 0.64$ & $4.72 \pm 0.51$ & 0.481 & 0.632 \\
\hline Leukocyte (x10 $\left.10^{9}\right)$ & $8.15 \pm 1.17$ & $7.96 \pm 1.34$ & 0.714 & 0.477 \\
\hline Time interval after stent/tube insertion (days) & $12.85 \pm 2.36$ & $11.92 \pm 2.05$ & 1.960 & 0.053 \\
\hline $\begin{array}{l}\text { Sex } \\
\text { Male } \\
\text { Female }\end{array}$ & $\begin{array}{l}36(73.47) \\
13(26.53)\end{array}$ & $\begin{array}{r}31(77.50) \\
9(22.50)\end{array}$ & 0.192 & 0.661 \\
\hline $\begin{array}{l}\text { Smoking } \\
\text { Yes } \\
\text { No }\end{array}$ & $\begin{array}{l}34(69.39) \\
15(30.61)\end{array}$ & $\begin{array}{l}29(72.50) \\
11(27.50)\end{array}$ & 0.103 & 0.748 \\
\hline $\begin{array}{l}\text { Alcohol consumption } \\
\text { Yes } \\
\text { No }\end{array}$ & $\begin{array}{r}40(81.63) \\
9(18.37)\end{array}$ & $\begin{array}{l}30(75.00) \\
10(25.00)\end{array}$ & 0.577 & 0.448 \\
\hline $\begin{array}{l}\text { Exercise habit } \\
\text { Yes } \\
\text { No }\end{array}$ & $\begin{array}{r}5(10.20) \\
44(89.80)\end{array}$ & $\begin{array}{c}3(7.50) \\
37(92.50)\end{array}$ & 0.197 & 0.657 \\
\hline $\begin{array}{l}\text { Ethnicity } \\
\text { Han } \\
\text { Minority }\end{array}$ & $\begin{array}{c}47(95.92) \\
2(4.08)\end{array}$ & $\begin{array}{c}37(92.50) \\
3(7.50)\end{array}$ & 0.485 & 0.486 \\
\hline $\begin{array}{l}\text { Pathological stage } \\
\text { Stage I-II } \\
\text { Stage III-IV }\end{array}$ & $\begin{array}{l}24(48.98) \\
25(51.02)\end{array}$ & $\begin{array}{l}22(55.00) \\
18(45.00)\end{array}$ & 0.320 & 0.572 \\
\hline $\begin{array}{l}\text { Lymphatic metastasis } \\
\text { Yes } \\
\text { No }\end{array}$ & $\begin{array}{l}17(34.69) \\
32(65.31)\end{array}$ & $\begin{array}{l}11(27.50) \\
29(72.50)\end{array}$ & 0.529 & 0.467 \\
\hline $\begin{array}{l}\text { Differentiation degree } \\
\text { Well-differentiated } \\
\text { Moderately differentiated } \\
\text { Poorly differentiated }\end{array}$ & $\begin{array}{l}27(55.10) \\
12(24.49) \\
10(20.41)\end{array}$ & $\begin{array}{r}20(50.00) \\
11(27.50) \\
9(22.50)\end{array}$ & 0.231 & 0.891 \\
\hline $\begin{array}{l}\text { Intestinal obstruction degree } \\
\text { Complete } \\
\text { Incomplete }\end{array}$ & $\begin{array}{l}32(65.31) \\
17(34.69)\end{array}$ & $\begin{array}{l}27(67.50) \\
13(32.50)\end{array}$ & 0.047 & 0.828 \\
\hline $\begin{array}{l}\text { Primary tumor } \\
\text { Colon cancer } \\
\text { Rectal cancer }\end{array}$ & $\begin{array}{l}21(42.86) \\
28(57.14)\end{array}$ & $\begin{array}{l}18(45.00) \\
22(55.00)\end{array}$ & 0.041 & 0.839 \\
\hline $\begin{array}{l}\text { Histological type } \\
\text { Adenocarcinoma } \\
\text { Mucinous carcinoma } \\
\text { Undifferentiated carcinoma }\end{array}$ & $\begin{array}{l}20(40.82) \\
19(38.78) \\
10(20.41)\end{array}$ & $\begin{array}{r}15(37.50) \\
14(35.00) \\
9(22.50)\end{array}$ & 0.136 & 0.934 \\
\hline $\begin{array}{l}\text { Lymph node dissection } \\
\text { Total mesangiectomy } \\
\text { Local excision }\end{array}$ & $\begin{array}{l}16(32.65) \\
33(67.35)\end{array}$ & $\begin{array}{l}14(35.00) \\
26(65.00)\end{array}$ & 0.054 & 0.816 \\
\hline
\end{tabular}


Table II. Open surgery rate comparison [n (\%)].

\begin{tabular}{lcccr}
\hline Factors & Stent group $(\mathrm{n}=49)$ & Catheter group $(\mathrm{n}=40)$ & $\chi^{2}$ test & P-value \\
\hline Open surgery rate & $2(4.08)$ & $3(7.50)$ & 0.485 & 0.486 \\
Incision infection & $3(6.12)$ & $2(5.00)$ & & \\
Anastomotic fistula & $1(2.04)$ & $2(5.00)$ & & \\
Lower extremity venous thrombosis & $1(2.04)$ & $0(0.00)$ & & \\
Abdominal infection & $2(4.08)$ & $2(5.00)$ & \\
Abdominal pain & $4(8.16)$ & $3(7.50)$ & 0.161 & \\
Fever & $3(6.12)$ & $4(10.00)$ & & \\
Incidence of adverse reactions $(\%)$ & 28.57 & 32.50 & \\
Hepatic metastases & $1(2.04)$ & $0(0.00)$ & & \\
Pulmonary metastasis & $0(0.00)$ & $1(2.56)$ & & \\
Brain metastases & $0(0.00)$ & $1(2.56)$ & & \\
Gastric metastasis & $1(2.04)$ & $0(0.00)$ & 5.037 \\
Local recurrence rate $(\%)$ & 4.26 & 5.13 & \\
\hline
\end{tabular}

group, $5.00 \%$ ( 2 cases) of patients had incision infection; $5.00 \%$ ( 2 cases) had anastomotic fistula; $5.00 \%$ ( 2 cases) had abdominal infection; $7.50 \%$ (3 cases) had abdominal pain; $10.00 \%$ (4 cases) had fever, and the incidence of adverse reactions was $32.50 \%$ (13 cases). There was no significant difference in the incidence of adverse reactions between the two groups ( $\mathrm{P}>0.05)$ (Fig. 1D and Table II).

Prognostic indicators. Eighty-six of 89 subjects were followed up successfully, and the success rate was $96.63 \%$. Two participants were lost to follow-up in the stent group and 1 case in the catheter group. There was no incision tumor implantation in either group. In the stent group, 2.13\% (1 case) of patients had hepatic metastasis, $2.13 \%$ (1 case) had gastric metastasis, and the local recurrence rate was $4.26 \%$ (2 cases); In the catheter group, $2.56 \%$ (1 case) of patients had pulmonary metastasis, $2.56 \%$ (1 1case) had brain metastasis, and the local recurrence rate was $5.13 \%$ ( 2 cases). There was no significant difference in local recurrence rate between the two groups $(\mathrm{P}>0.05)$ (Table II).

\section{Discussion}

There is a high probability of intestinal obstruction in patients with colorectal cancer which may lead to the reduction of resection rate, increase of intestinal orifice rate, and a significant increase in risk of death after surgery (14). Because of intestinal obstruction, preoperative bowel preparation is not possible for colorectal cancer patients. Patients were usually performed proximal obstruction of intestinal fistula first and then resection surgery, and an intestinal stoma reversion was conducted after the surgery $(15,16)$. Multiple trauma surgery causes more damage to patients who are already suffering from cancer, and the sequelae of trauma surgery multiplies $(17,18)$. With the widespread use of endoscopic stents and trans-anal ileus catheterization in recent years, the clinical efficacy of patients with obstructive colorectal cancer has significantly improved. At present, endoscopic stent and trans-anal ileus catheterization combined with laparoscopic surgery in the treatment of obstructive colorectal cancer has achieved significant research results, which has great value in improving the success rate of the surgery of obstructive colorectal cancer. Through endoscopic stent and trans-anal ileus catheterization, the intestinal obstruction of colorectal cancer patients can be relieved by non-operative method $(19,20)$, which makes the tumor resection surgery standard, and not only greatly increase the success rate of resection surgery, but also effectively improve the prognosis of patients.

The clinical value of endoscopic stent and trans-anal ileus catheterization combined with neoadjuvant chemotherapy in laparoscopic resection of obstructive colorectal cancer was compared in this study, and the results showed that the intestinal preparation time in the stent group was shorter than that in the catheter group, which indicated that endoscopic stent was more effective than trans-anal ileus catheterization in relieving intestinal obstruction. The reason might be that the small diameter of trans-anal ileus catheter, the poor effect of defecation rate, disunited defecation of patients and higher stool hardness in some patients can cause intestinal catheter obstruction, resulting in the intestinal preparation time in the catheter group being longer than the stent group. However, the surgical duration and the intraoperative blood loss in the stent group were higher than those in the catheter group, which suggested that trans-anal ileus catheter had higher surgical safety than endoscopic stent in patients with obstructive colorectal cancer. The reason was that intestinal stents might lead to intestinal perforation and bleeding, and stent displacement might lead to abnormal intestinal metabolism in patients (18). During the operation, the doctor not only needs to remove the tumor focus completely, but must also remove the implanted stent, which would take more time and blood loss during the surgery than in the catheter group. Catheterization could indirectly improve intestinal edema and intestinal wall blood flow in patients (21). The catheter is removed preoperatively, allowing the doctor to focus on the removal of the tumor during the surgery. Smooth environment 
of the patient's intestine is beneficial to surgery. There was no significant difference in open surgery rate, postoperative adverse reactions, exhaust time and prognosis between the two groups, which indicated that trans-anal ileus catheter and endoscopic stent had high application value in patients with obstructive colorectal cancer. Because of the presence of intestinal obstruction, patients with obstructive colorectal cancer usually need to wash their intestines before and after surgery the intestine is the largest digestive organ in the human body, which contains a large amount of feces and bacteria. Once the bacteria invade the body during surgery, it is easy to cause infection, which directly results in the decrease of the immune function of the patients, causes postoperative complications and affects the prognosis. The intestinal function of patients can be improved effectively with trans-anal ileus catheter and endoscopic stent, and combined with intravenous parenteral nutrition intervention can obtain effective nutritional support after surgery, improve the stability and balance of intestinal tract, reduce the occurrence of postoperative adverse reactions, and greatly improve the prognosis of patients.

The purpose of this study was to compare the clinical efficacy of trans-anal ileus catheter and endoscopic stent in patients with obstructive colorectal cancer, but there were still some shortcomings due to the limited experimental conditions. The number of subjects studied was small and the population was relatively uniform. The materials of intestinal obstruction stents are varied. The stents used in this study were all made of metal materials, which did not exclude the possible differences in the efficacy of other materials (such as stainless steel and nickel-titanium alloy). A longer-term follow-up survey of the subjects in this study will be conducted.

In conclusion, intestinal metal stent implantation can effectively relieve intestinal obstruction, while trans-anal ileus catheter offers higher safety in laparoscopic surgery. Their combination with neoadjuvant chemotherapy and laparoscopic surgery for obstructive colorectal cancer has high value and good clinical effect. The best treatment plan should be selected according to the patient's condition.

\section{Acknowledgements}

Not applicable.

\section{Funding}

This study was supported by Taizhou Science and Technology Project. (no. 1701KY07).

\section{Availability of data and materials}

The datasets used and/or analyzed during the present study are available from the corresponding author on reasonable request.

\section{Authors' contributions}

LY and WM designed the study and drafted the manuscript. LY, MW and RZ acquired the data. WM, TB and SZ analyzed the data and revised the manuscript. All the authors read and approved the final manuscript.

\section{Ethics approval and consent to participate}

The study was approved by the Ethics Committee of Taizhou Hospital of Zhejiang Province, Wenzhou Medical University (Taizhou, China). Patients who participated in this research, signed an informed consent and had complete clinical data.

\section{Patient consent for publication}

Not applicable.

\section{Competing interests}

The authors declare that they have no competing interests.

\section{References}

1. Siegel RL, Miller KD, Fedewa SA, Ahnen DJ, Meester RG, Barzi A and Jemal A: Colorectal cancer statistics, 2017. CA Cancer J Clin 67: 177-193, 2017.

2. Arnold M, Sierra MS, Laversanne M, Soerjomataram I, Jemal A and Bray F: Global patterns and trends in colorectal cancer incidence and mortality. Gut 66: 683-691, 2017.

3. Takahashi H, Okabayashi K, Tsuruta M, Hasegawa H, Yahagi M and Kitagawa Y: Self-expanding metallic stents versus surgical intervention as palliative therapy for obstructive colorectal cancer: A meta-analysis. World J Surg 39: 2037-2044, 2015.

4. Atsushi I, Mitsuyoshi O, Kazuya Y, Syuhei K, Noriyuki K, Masashi M, Akira W, Kentaro S, Nobuyuki K, Natsuko S, et al: Long-term outcomes and prognostic factors of patients with obstructive colorectal cancer: A multicenter retrospective cohort study. World J Gastroenterol 22: 5237-5245, 2016.

5. Kegler MC, Beasley DD, Liang S, Cotter M, Phillips E, Hermstad A, Williams R, Martinez J and Riehman K: Using the consolidated framework for implementation research to understand safety net health system efforts to increase colorectal cancer screening rates. Health Educ Res 33: 315-326, 2018.

6. Baer C, Menon R, Bastawrous S and Bastawrous A: Emergency presentations of colorectal cancer. Surg Clin North Am 97: 529-545, 2017.

7. Khoo E, O'Neill S, Brown E, Wigmore SJ and Harrison EM: Systematic review of systemic adjuvant, neoadjuvant and perioperative chemotherapy for resectable colorectal-liver metastases. HPB (Oxford) 18: 485-493, 2016.

8. Kwak MS, Kim WS, Lee JM, Yang DH, Yoon YS, Yu CS, Kim JC and Byeon JS: Does stenting as a bridge to surgery in left-sided colorectal cancer obstruction really worsen oncological outcomes? Dis Colon Rectum 59: 725-732, 2016.

9. Haraguchi N, Ikeda M, Miyake M, Yamada T, Sakakibara Y, Mita E, Doki Y, Mori M and Sekimoto M: Colonic stenting as a bridge to surgery for obstructive colorectal cancer: Advantages and disadvantages. Surg Today 46: 1310-1317, 2016.

10. Cézé N,Charachon A, LocherC, Aparicio T, Mitry E, Barbieux JP, Landi B, Dorval E, Moussata D and Lecomte T: Safety and efficacy of palliative systemic chemotherapy combined with colorectal self-expandable metallic stents in advanced colorectal cancer: A multicenter study. Clin Res Hepatol Gastroenterol 40: 230-238, 2016.

11. Miłek T and Ciostek P: Implantation of a new enteral stent in obstructive colorectal cancer using interventional radiology in patients over 70 years of age. Wideochir Inne Tech Malo Inwazyjne 10: 155-160, 2015.

12. Kim EJ and Kim YJ: Stents for colorectal obstruction: Past, present, and future. World J Gastroenterol 22: 842-852, 2016.

13. De Rosa M, Pace U, Rega D, Costabile V, Duraturo F, Izzo P and Delrio P: Genetics, diagnosis and management of colorectal cancer (Review). Oncol Rep 34: 1087-1096, 2015.

14. Mattar RE, Al-Alem F, Simoneau E and Hassanain M: Preoperative selection of patients with colorectal cancer liver metastasis for hepatic resection. World J Gastroenterol 22: 567-581, 2016.

15. Enomoto T, Saida Y, Takabayashi K, Nagao S, Takeshita E, Watanabe R, Takahashi A, Nakamura Y, Asai K, Watanebe M, et al: Open surgery versus laparoscopic surgery after stent insertion for obstructive colorectal cancer. Surg Today 46: 1383-1386, 2016. 
16. Yamada T, Okabayashi K, Hasegawa H, Tsuruta M, Yoo JH Seishima R and Kitagawa Y: Meta-analysis of the risk of small bowel obstruction following open or laparoscopic colorectal surgery. Br J Surg 103: 493-503, 2016.

17. Cetinkaya E, Dogrul AB and Tirnaksiz MB: Role of self expandable stents in management of colorectal cancers. World J Gastrointest Oncol 8: 113-120, 2016.

18. Zullig LL, Smith VA, Jackson GL, Danus S, Schnell M, Lindquist J, Provenzale D, Weinberger M, Kelley MJ and Bosworth HB: Colorectal cancer statistics from the veterans affairs central cancer registry. Clin Colorectal Cancer 15: e199-e204, 2016.

19. Shimizu H, Yamazaki R, Ohtsuka H, Osaka I, Takuma K and Morita Y: Feasibility of laparoscopic surgery after stent insertion for obstructive colorectal cancer. Asian J Endosc Surg 11: $118-122,2018$.
20. Park SJ, Lee KY, Kwon SH and Lee SH: Stenting as a bridge to surgery for obstructive colon cancer: Does it have surgical merit or oncologic demerit? Ann Surg Oncol 23: 842-848, 2016.

21. Zhang N, Zhou ZL and Xie JL: Application of transanal ileus tube in acute obstructive left-sided colorectal cancer. Int J Clin Exp Med 8: 14024-14029, 2015.

This work is licensed under a Creative Commons Attribution-NonCommercial-NoDerivatives 4.0 International (CC BY-NC-ND 4.0) License. 